\title{
A Framework for Situational Web Methods Engineering
}

\author{
Sebastian Lahajnar \\ Pris inženiring d.o.o. Ljubljana, Slovenia \\ sebastian. lahajnar@pris-inz.si
}

\begin{abstract}
In the past ten years many web application development methods with their own or from other methods borrowed models, techniques and activities were proposed in literature. Each of these methods is appropriate for building several types of web applications on different modeling levels and project phases. It's unlikely that a single method will ever be capable to cover all aspects of web application development. The most appropriate approach for web application projects is a construction of an organization-specific base method with the use of reusable method fragments (components) and the adaptation of the base method in order to support specific project characteristics. For this purpose, the basis for an appropriate method engineering framework is proposed, which includes a process for method construction and a repository for methods, method components, configurations, rules and development situations characteristics storage.
\end{abstract}

\section{Introduction}

Nowadays, web applications have an important role in a development of information systems. As Kiely and Fitzgerald state [8], 33\% of development projects can be classified as E-Commerce, $7 \%$ involves building internet sites, while only $25 \%$ involves traditional application development (payroll systems etc.). Comparing two empirical researches ([8], [7]) of the information systems development environment it can be noticed, that the use of methods for software development is increasing (from $40 \%$ in 1998 to $62 \%$ in 2002). Consequently, also the increasing interest in using and building methods, techniques and tools, designed specially for web application development is understandable.

In the past ten years many web application development methods based on traditional and object models, languages and processes such as ER, UML and Unified Process were proposed. With regard to their origins and purposes, they can be categorized as data, hypertext, object or process oriented [11]. The origin, initial scope and later extensions influence the usability of methods for various types of web applications (portals, transactional, document systems etc.) on different levels of modeling (conceptual, logical) and in different phases (requirements gathering, analysis, design, realization etc.). In this sense, WebML [5] is suitable for the specification of complex data intensive web applications at the conceptual level, SOHDM [14] emphasizes a process driven web applications development, Conallen [6] proposes a development process based on RUP and advocates the importance of modeling concrete products in 
the design phase with the use of UML WAE profile, UWE [13] focuses on the navigation and presentation aspects of web applications, the primary purpose of WebSA [15] method is to define the architectural view of web applications etc.

\section{The Problem Domain}

From the analysis of current web development methods it is possible to deduce, that their activities and models are well suited for describing navigational, presentational and architectural views on different levels. For the description of data view at the conceptual level, web development methods use traditional models and techniques such as ER model and UML class diagram. Web development methods mostly don't concern with activities for business modeling, project management, testing, maintenance, portfolio management etc., which are part of traditional development processes. On the other hand, traditional development processes and modeling languages do not comprehend adequate activities and modeling techniques for the description of web applications particularity, such as navigation and presentation, and particularity of other modeling domains as for example business process modeling and data modeling. The solution is provided by number of existing UML profiles and other modeling techniques, which can be included in a development process in order to improve a reliability of developed products.

Web application development in the context of current organization's characteristics, complexity of the solution, the size of the project and other factors, may include a number of more or less important activities with the final goal to deploy a working web application. The structure, activities and produced artifacts must be adapted to concrete situations what means, that the method can not be fully defined in advance. Nevertheless, it is recommended to develop a base organization-specific method, founded on experiences acquired in previous projects, good practices and organization culture, which can be used as a reference for a construction of adapted methods for specific project situations. A base method as all other adapted methods must be composed from a set of best, autonomous and reusable fragments, which will provide good foundations for successful project progress in given circumstances. Since such fragments belong to different software development methods and processes, they have to be re-engineered in order to be successfully included in the new method in construction. Following the reflection in previous paragraphs, two theses are defined: 1) it's unlikely that a single method will ever be capable to cover all aspects of web application development. 2) the most appropriate approach for web application development projects is a construction of organization-specific base method on the basis of reusable method fragments stored in a repository, which is then tailored to support specific project needs.

\section{The Aims and Objectives of the Research}

The main objective of the research is to build a common framework for web application method engineering. In this context, many traditional and new approaches for situational method engineering have been already investigated. The effort resulted in 
the definition of the framework foundations with the corresponding process of reengineering parts of widely recognized methods in method fragments, constructing base method with method fragments assembly and tailoring base method to project specific demands.

The proposed framework is supposed to include the most important activities, models and techniques which nowadays are used in information systems development, as also their extensions in the field of web engineering, business processes and databases, with the emphasis on modern object oriented development processes and the UML as a main modeling language.

\section{The Solution}

The discipline to design, construct and adapt methods, techniques and tools for information systems development is called method engineering, while its specific direction, situational method engineering, deals with a development of specific methods, tailored for concrete organization or project circumstances [2]. The construction of new methods is founded on standardized assets called method fragments, defined as coherent pieces of information systems development methods. Brinkkemper [3] classifies method fragments according to three dimensions: perspective (product, process), abstraction (conceptual, technical) and granularity (method, stage, model, diagram and concept). The process and the product view of the method fragment are inseparable interrelated as products are both results and inputs of the method's prescribed actions.

Instead of method fragments, Wistrand [19] proposes the concept of method component, defined as a self-contained part of the system engineering method expressing the process of transforming input artifacts into defined target artifacts and a rationale for such a transformation. In this way, a method component is a special case of a method fragment with the following characteristics: self-contained, internal consistency and coherency, rationality and applicability. Two views describes each method component: the internal view addresses the internal structure of the component while the external view focuses on its relationships with other components which all together contribute to the overall method goal. For our purpose, the concept of method component have been chosen as the foundation for the development of the web methods engineering framework as it emphasizes higher level of granularity, fragments independence and fragments interconnection through interfaces.

The process of building the method base is based on the re-engineering of existing methods or their individual fragments and includes the following steps [17]: defining or reconstructing the initial method process model, identifying method components and defining method components. The construction of the method base is a continuous process at the beginning of which the method base is populated with an initial set of method components. The initial set is further enhanced during the process of building the base method and it's adaptation to specific projects.

After the initial set of method components has been defined, the construction of the base method takes place with the use of the assembly strategy following the next three steps [18]: project characterization, method fragments selection and method fragments assembly. Two basic strategies are defined for method fragments assembly 


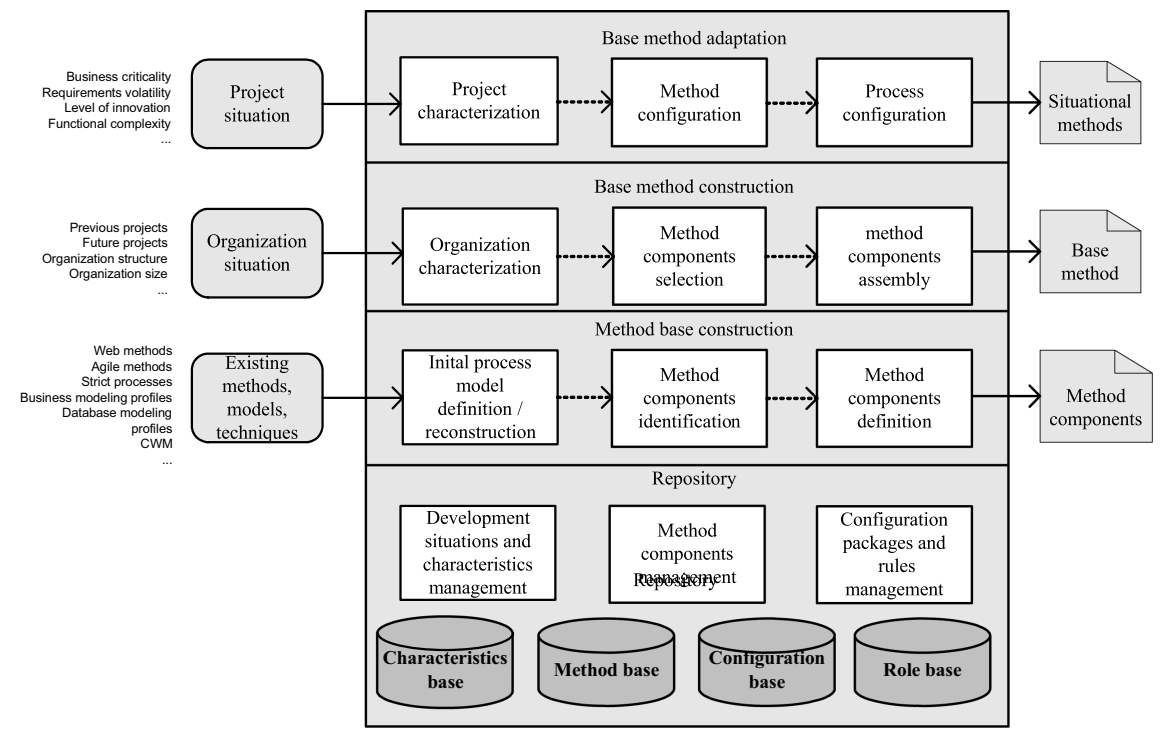

Fig. 1. The structure of the framework

[17]: association and integration. The association strategy is appropriate in a case, when assembled method fragments don't have common elements, while the integration strategy is relevant for the assembly of methods with common engineering objectives, but different ways to achieve them.

The combination of two strategies, method configuration [12] and process configuration [1] respectively, has been chosen for the adaptation of the base method to specific projects. The reusable concepts, a configuration package and a configuration template form the foundation of this strategy. The configuration package is defined as a configuration of the base method suitable for one specific characteristic's value and the configuration template is defined as a combined method configuration for a set of recurrent project characteristics [12]. The process configuration strategy supplements method configuration by enabling the definition of alternative paths in the context of configuration packages. The process configuration is performed on the basis of a set of rules for structural and process restrictions, depending on the specific circumstances.

\section{Related Work}

Works resembling the framework proposed in this paper comes from two disciplines: web and method engineering. In the area of web engineering, Koch [13] defines a metamodel for UWE approach, which describes the concepts of the navigation and presentation model. This and other proposed metamodels for various web methods could be seen as a foundation for one or more method components, focusing on the navigational and presentational characteristics of the web application. Caceres [4] advocates MIDAS, a model-driven methodology for web IS development in which he 
incorporates several models and diagram technique from other web and non web methods for structural PIMs and PSMs. Moreno [16] on the other hand, distinguishes three main PIMs: user interface, business logic and data. For each of them, he proposes a set of feasible models with appropriate concepts. Both approaches share many similarities with the proposed framework, as many different models and technique from different fields are used, and there's also some possibility to tailor the two methods to suit the project's specific needs. However, the framework proposed in this paper takes a broader perspective to web application development as it enables the integration of almost any desired and useful technique, model, concept, activity or whole method, as long as it's adequate represented following the rules of the method components metamodel (internal and external view).

From the sphere of method engineering, the OPF framework [9] should be mentioned, which besides the common set of elements for the software development process, incorporates also some web specific activities, tasks and roles. Nevertheless, OPF doesn't address adequately all web applications specific aspects such as navigation and presentation and furthermore, for each project a completely new instance of the OPF metamodel has to be instantiated, what increases project overall cost and duration.

\section{Future Work and Conclusions}

A proper metamodel for the method base as a combination of many existing metamodels (method components [19], SMSDM [10], method configuration [12] etc.) is currently under development. After that an already identified set of valuable activities, work products, models and techniques from different software engineering fields (web, database, business process etc.) will be further re-engineered in method components in concordance to the metamodel specified. Based on the developed set of method components, a new organization-specific method for web application development will be constructed with the application of the assembly strategy on the basics of defined components interfaces. Finally, the proposed framework and the base method will be validated on the concrete project, involving a development of many web portals for different faculties in Slovenia which is already in progress at the time of this writing. The new method for web application development will be evaluated against the current approach on the basis of a set of predefined criteria, using a questionnaire and performing interviews with project team members.

\section{References}

1. Bajec, M., Vavpotič, D., Krisper, M.: An approach for creating project-specific software development methodologies. In: Internet and information technology in modern organizations, IBIMA, pp. 1-15 (2005)

2. Brinkkemper, S.: Method engineering: Engineering of information systems development methods and tools. Information and Software Technology 38(4), 275-280 (1996)

3. Brinkkemper, S., Saeki, M., Harmsen, F.: Meta-modelling based assembly techniques for situational method engineering. Information Systems 24 (1999), 209-228 (1999)

4. Cáceres, P., Marcos, E., Vela, B.: A MDA-Based Approach for Web Information System. In: Workshop in Software Model Engineering, WisME (2004) 
5. Ceri, S., Fraternali, P., Matera, M.: Conceptual Modeling of Data-Intensive Web Applications. IEEE Internet Computing 6(4), 20-30 (2002)

6. Conallen, J.: Building Web Applications with UML, 2nd edn. Addison-Wesley, London, UK (2003)

7. Fitzgerald, B.: An empirical investigation into the adoption of systems development methodologies. Information and Management 34(6), 317-328 (1998)

8. Kiely, G., Fitzgerald, B.: An investigation of the information systems development environment: the nature of development life cycles and the use of methods. In: Eighth Americas Conference on Information Systems, Baylor, pp. 1289-1296 (2002)

9. Haire, B., Lowe, D., Henderson-Sellers, B.: Supporting Web development in the OPEN process: Additional roles and techniques. In: Bellahsène, Z., Patel, D., Rolland, C. (eds.) OOIS 2002. LNCS, vol. 2425, pp. 82-94. Springer, Heidelberg (2002)

10. Henderson-Sellers, B., Gonzalez-Perez, C.: A comparison of four process metamodels and the creation of a new generic standard. Information \& Software Technology 47(1), 49-65 (2005)

11. Kappel, G.: Web Engineering, The Discipline of Systematic Development of Web Applications. John Wiley \& Sons, West Sussex, England (2006)

12. Karlsson, F., Ågerfalk, P.J.: Method configuration: adapting to situational characteristics while creating reusable assets. Information and Software Technology 46, 619-633 (2004)

13. Koch, N., Kraus, A.: Towards a Common Metamodel for the Development of Web Applications. In: Lovelle, J.M.C., Rodríguez, B.M.G., Gayo, J.E.L., Ruiz, M.d.P.P., Aguilar, L.J. (eds.) ICWE 2003. LNCS, vol. 2722, pp. 497-506. Springer, Heidelberg (2003)

14. Lee, H., Lee, C., Yoo, C.: A scenario-based object-oriented methodology for developing hypermedia information systems. In: Sprague, R. (eds.) Proceedings of 31 st Annual Conference on Systems Science (1998)

15. Meliá, S., Gómez, J., Koch, N.: Improving Web Design Methods with Architecture Modeling. In: Bauknecht, K., Pröll, B., Werthner, H. (eds.) EC-Web 2005. LNCS, vol. 3590, pp. 53-64. Springer, Heidelberg (2005)

16. Moreno, N., Vallecillo, A.: A Model-based Approach for Integrating Third Party Systems with Web Applications. In: Lowe, D.G., Gaedke, M. (eds.) ICWE 2005. LNCS, vol. 3579, pp. 441-452. Springer, Heidelberg (2005)

17. Ralyte, J., Rolland, C.: An Assembly Process Model for Method Engineering. In: Dittrich, K.R., Geppert, A., Norrie, M.C. (eds.) CAiSE 2001. LNCS, vol. 2068, Springer, Heidelberg (2001)

18. Ralyté, J., Rolland, C.: An approach for method reengineering. In: Kunii, H.S., Jajodia, S., Sølvberg, A. (eds.) ER 2001. LNCS, vol. 2224, Springer, Heidelberg (2001)

19. Wistrand, K., Karlsson, F.: Method Components - Rationale Revealed. In: The 16th International Conference on Advanced Information Systems Engineering, Riga, Latvia (2004) 